\title{
KINETIC GRAVITY SEPARATION
}

\author{
LAURENS VAN KOOY, MARIEKE MOOIJ and PETER REM* \\ Delft University of Technology, Mijnbouwstraat 120, 2628 RX Delft, The Netherlands
}

(Received 16 March 2004; In final form 8 April 2004)

\begin{abstract}
Separations by density, such as the separation of non-ferrous scrap into light and heavy alloys, are often realized by means of heavy media. In principle, kinetic gravity separations in water can be faster and cheaper, because they do not rely on suspensions or salt solutions of which the density must be tightly controlled. Test results of a prototype kinetic gravity separator in combination with suitable size classification techniques show promising results for particle sizes between 2 and $10 \mathrm{~mm}$.
\end{abstract}

Keywords: Gravity separation; Separation by density; Non-ferrous metals; Plastics

\section{INTRODUCTION}

Separations on density are often effected by a sink-float in heavy media or in salt solutions [1,2]. One of the main problems and also an important cost factor is that the density of the separation medium should be tightly controlled. Inevitably, part of the medium is carried away with the products, and often this material needs to be recovered after the washing step. Another drawback of sink-float separations, especially in dense suspensions, is that the kinetics of the separation is relatively slow for small particles. Sometimes, sink-floaters of non-ferrous metal concentrates push too much aluminium into the heavy non-ferrous product in attempts to combine a high feed rate with a wide range of particle sizes.

Kinetic gravity separation in water is a potentially cheaper alternative for mixtures with small particle sizes. In this kind of separation, the particles are separated on the basis of differences in their terminal velocity. Since the terminal velocity of a particle depends not only on its density but also on its size and shape, the effect of the latter two parameters must somehow be suppressed. Existing separators that are based on terminal velocity, such as jigs [1,2], deal with the adverse effects of particle size variations in the feed by operating at relatively high solids concentrations and by limiting the ratio of maximum to minimum particle size. Jigs have been used for both metals and plastics scrap [3] but the fluidization and mobility of scrap particles are not optimal, since some of these particles, in particular flat pieces and wires,

*Corresponding author. E-mail: p.c.rem@citg.tudelft.nl 
have strongly non-granular shapes. For this reason, an alternative kinetic gravity separator was designed [4] in which the solids concentration is low and particles are separated strictly on terminal velocity. Materials of different sizes can be fed at different points in order to deal with variations of particle size. One of the problems is to combine kinetic gravity separation with a suitable classification technique. In fact, finding suitable techniques for plastics and non-ferrous scrap has been a major part of the present research.

\section{KINETIC GRAVITY SEPARATOR}

The concept of separation on terminal velocity is that particles are fed at some point in a stream of fluid that is flowing in a horizontal direction. Quickly after being released, the particles reach their terminal velocity with respect to the fluid. From this point on, particles have a horizontal velocity component that is the same as that of the fluid and a vertical component that depends on their density, size and shape. At some level with respect to the feeding point, the particles are collected in a series of compartments that are aligned in the direction of the flow (see Fig. 1(a)). Particles with a high vertical speed end up in the compartments close to the feeding point, whereas particles settling at a lower speed are collected further downstream. From an engineering point of view, it is attractive to make the fluid flow in a closed loop (Fig. 1(b)) because it is relatively easy to create a rotational flow with little turbulence.

The prototype separator essentially consists of two coaxial cylindrical shells of different diameters that are connected by some 50 radial fins, spaced at distances of about $5 \mathrm{~cm}$ (see Fig. 1(b)). The structure is submerged in a fluid and rotates around its vertical axis so as to force the fluid between the shells to move at a constant angular velocity. The main reason for spacing the fins so closely is to prevent the particles from any significant horizontal translations with respect to the medium in the angular direction. If scrap particles are allowed to move freely within a fluid, their motion has a considerable stochastic horizontal component, due to the effect of shape. Such a stochastic behaviour destroys the sharpness of separation and therefore the particles are confined between the closely spaced fins. Particles are fed from the top (if heavier

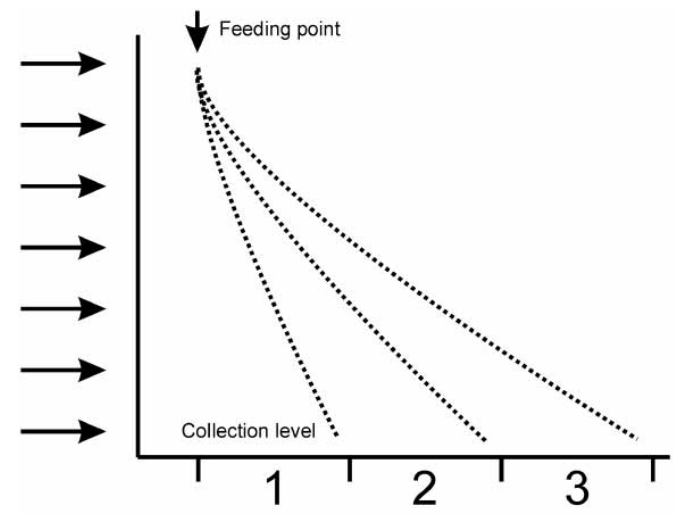

(a)

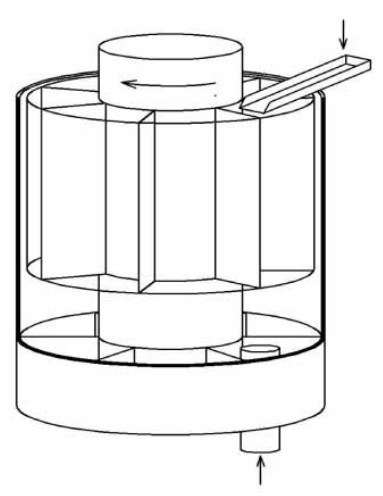

(b)

FIGURE 1 Principle of kinetic gravity separation: (a) linear fluid flow; (b) rotational fluid flow. 


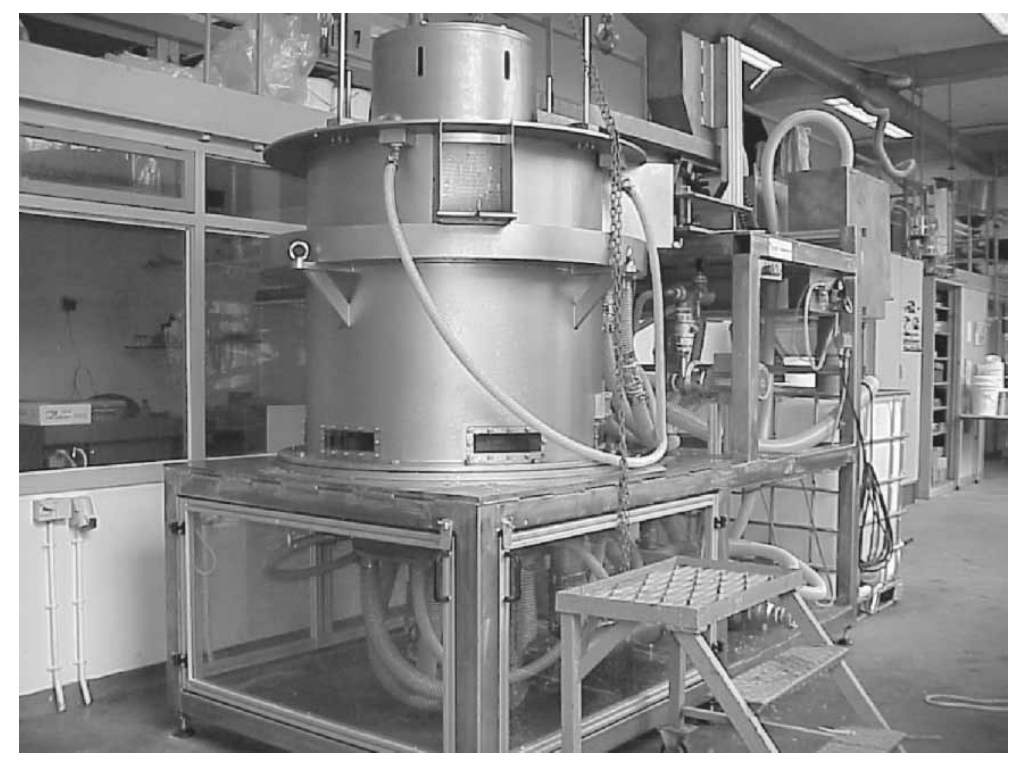

FIGURE 2 Experimental set-up: kinetic gravity separator (the outer cylinder shell is $1 \mathrm{~m}$ high).

than the medium) or from the bottom (if lighter than the medium) and collected in six compartments below the separation zone or in three compartments at the surface. For any given particle, the angle between the points of feeding and collection is directly proportional to the amount of time spent in the separation zone between the shells and consequently inversely proportional to the particle's terminal velocity. By feeding particles of different sizes at different feeding points, particles of similar densities are collected in the same product compartment. Figure 2 shows a photograph of the actual machine. The tubes extending from the bottom compartments are powered by a water jet to remove the metal and plastics products from the machine to screens at the back.

\section{THEORY}

The terminal velocity $v$ of a particle in a fluid results from the balance of drag, gravity and buoyancy:

$$
\left(\rho_{s}-\rho\right) V g=C \frac{1}{2} \rho v^{2} A
$$

where $g$ is the acceleration of gravity, $\rho_{s}$ and $\rho\left(\mathrm{kg} / \mathrm{m}^{3}\right)$ are the densities of the particle and the fluid, respectively, $V$ and $A$ are the volume and the cross-sectional area of the particle, and $C$ is the coefficient of drag, which is a function of the shape of the particle and the Reynolds number Re of the flow around the particle:

$$
\operatorname{Re}=\frac{\rho v D}{\eta}
$$




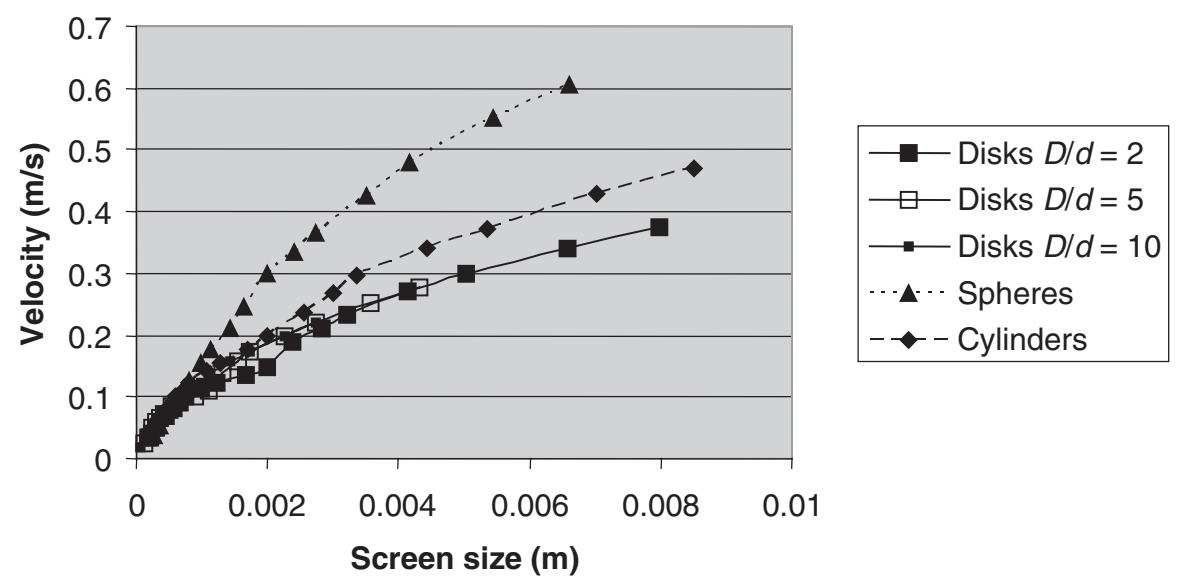

FIGURE 3 Theoretical terminal velocity of aluminium particles as a function of screen size.

$D$ is the characteristic dimension (diameter) of the particle and $\eta(\mathrm{kg} / \mathrm{sm})$ is the dynamic viscosity of the fluid. The drag coefficient is documented as a function of Re for particles with elementary shapes, such as spheres, cylinders and circular disks and a wide range of Reynolds numbers $\left(10^{-3}<\operatorname{Re}<10^{6}\right)$ [5]. This means that for these particle shapes, both the particle diameter $D$ and the corresponding terminal velocity $v$ can be related directly to Re:

$$
\begin{gathered}
D=\sqrt[3]{\frac{s \eta^{2} C(\mathrm{Re}) \mathrm{Re}^{2}}{2 \rho\left(\rho_{s}-\rho\right) g}} \\
v=\frac{\eta \operatorname{Re}}{\rho D}
\end{gathered}
$$

The shape parameter $s$ is equal to $D / d$ for a circular disk of thickness $d$, whereas it is equal to 1.5 for a sphere and equal to $4 / \pi$ for a cylinder. Note that the use of Eqs. (3) and (4) has the advantage that the results are not limited to a particular flow regime (e.g. laminar or turbulent).

Figure 3 shows a plot of the expected terminal velocity of granular, wire-shaped and flat aluminium scrap particles versus the screen size on a bar screen, based on the formulae for spheres, cylinders and disks given above. The theoretical screen size was taken to be $D$ for spheres and cylinders and $1.5 d$ for disks (to account for curved surfaces). The plot shows that the screen size on a bar screen is expected to correlate well with terminal velocity, except for granular particles.

\section{EXPERIMENTS WITH NON-FERROUS SCRAP}

A series of experiments were performed on non-ferrous scrap particles in the range of $2-10 \mathrm{~mm}$ to check whether a simple theory based on three perfect shapes would account for the observed terminal velocities of scrap particles in a tube of 
$50 \mathrm{~mm}$ internal diameter filled with water. The scrap contained aluminium and heavy non-ferrous metals, such as (alloys of) copper, zinc and lead (see Table I). Prior to the experiment, the scrap particles were screened at 2, 4, 6, 8 and $10 \mathrm{~mm}$ on a bar screen, and the resulting fractions were separated in a salt solution at $3000 \mathrm{~kg} / \mathrm{m}^{3}$ and hand-sorted to yield fractions of aluminium and heavy non-ferrous metals consisting of granular, wire-shaped or flat particles in four size ranges: $-4+2 \mathrm{~mm},-6+4 \mathrm{~mm}$, $-8+6 \mathrm{~mm}$ and $-10+8 \mathrm{~mm}$. Figure 4 shows the cumulative distribution of the terminal velocity for the $-8+6 \mathrm{~mm}$ heavy non-ferrous particles as a typical example. Results for granular particles are indicated by dotted lines, wires by dashed lines and flat pieces by solid lines. Straight lines have been drawn into the plot to indicate the expected distributions for the various shape classes on the basis of theoretical minimum and maximum velocities of spheres, cylinders and disks. The results show that the theory gives indicative values for wires and flat pieces, but also that granular particles do not settle as fast as would be expected from the theory for spheres. This means that the terminal velocity distributions for the various shapes differ less than would be expected on the basis of theory. It is also shown, however, that the actual distributions for individual shape categories are wider than expected on the basis of perfect shapes. In other words, the variations in shape within a particular shape class have a considerable effect on the terminal velocity. Figure 5 shows the entire range of terminal velocity distributions for all fractions, with aluminium indicated by white and heavy non-ferrous by black

TABLE I Material densities of metals and alloys found in $2-10 \mathrm{~mm}$ non-ferrous scrap

\begin{tabular}{lr}
\hline & $\rho_{s}\left(\mathrm{~kg} / \mathrm{m}^{3}\right)$ \\
\hline Aluminium & 2700 \\
Copper & 8900 \\
Brass & 8500 \\
Zinc & 7100 \\
Lead & 11400 \\
Solder & 8900 \\
\hline
\end{tabular}

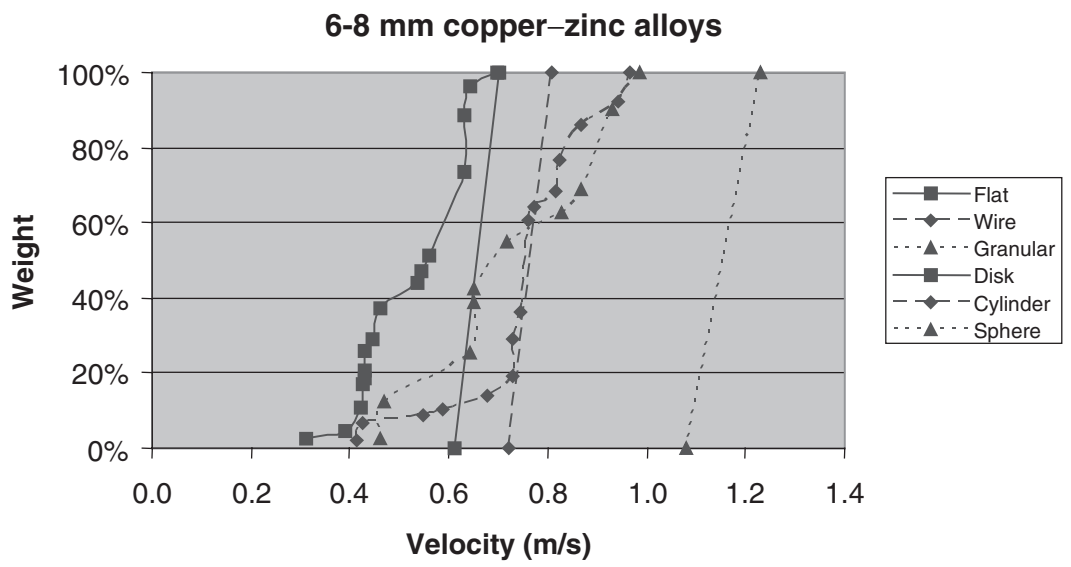

FIGURE 4 Actual versus predicted distributions of terminal velocity for various particle shapes. (Note that straight lines are theoretical distribution curves.) 

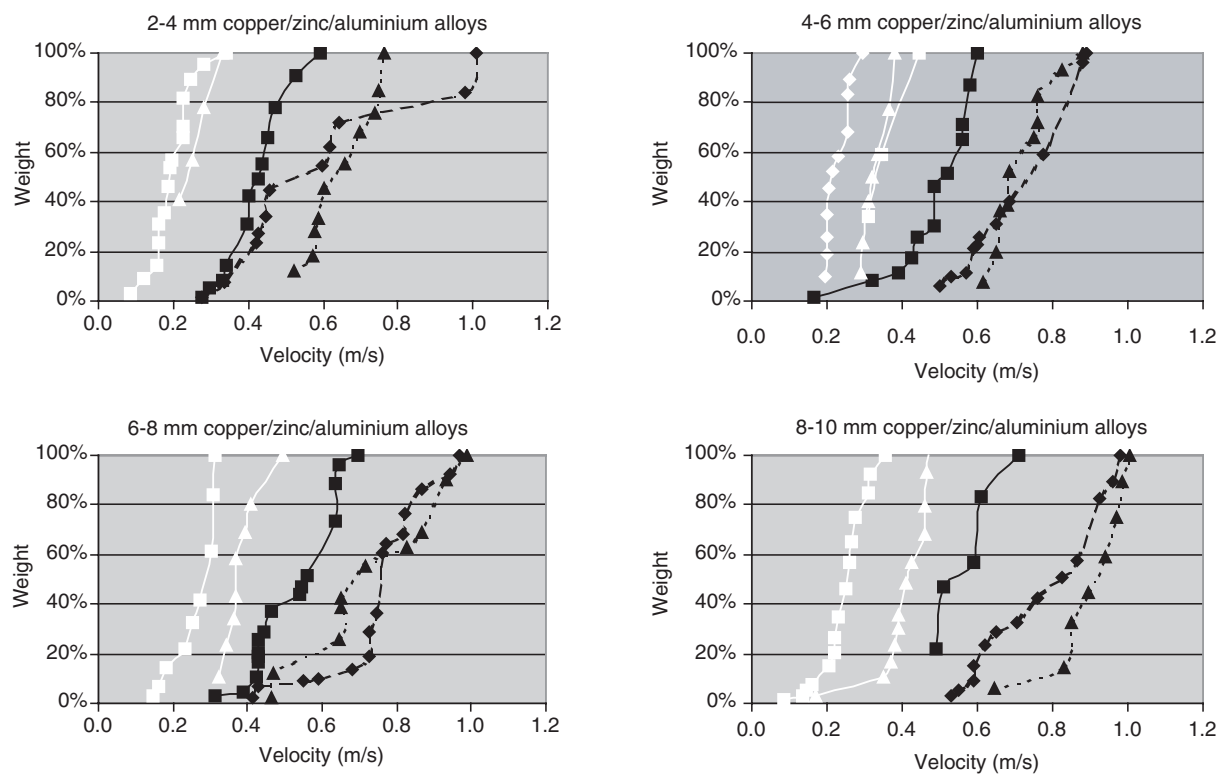

FIGURE 5 Actual distributions of terminal velocities for scrap particles in several size ranges. Light alloys (aluminium) are indicated in white, and heavy alloys (copper, zinc) are shown in black (the meaning of the marks is the same as in Fig. 4).

TABLE II Analysis of feed and products of the separation of the bottom ash concentrate

\begin{tabular}{lcccc}
\hline & $A l(\mathrm{~kg})$ & $\mathrm{Zn} / \mathrm{Cu}(\mathrm{kg})$ & Stone $(\mathrm{kg})$ & Tot $(\mathrm{kg})$ \\
\hline Feed & 8.0 & 9.8 & 62.2 & 80.0 \\
Compartment 1 & 0.2 & 9.9 & 0.9 & 11.0 \\
Compartment 2 & n.m. & 0.2 & n.m. & 65.3 \\
\hline
\end{tabular}

curves. The results suggest that non-ferrous scrap ranging from 2 to $10 \mathrm{~mm}$ can be separated into aluminum and heavy non-ferrous on the basis of terminal velocity, using a cut varying from about $0.35 \mathrm{~m} / \mathrm{s}$ at the small size limit to about $0.45 \mathrm{~m} / \mathrm{s}$ at the large size limit.

In order to test the separation in practice, a sample of about $80 \mathrm{~kg}$ of a $-6+2 \mathrm{~mm}$ non-ferrous concentrate obtained from bottom ash was separated with the prototype separator at a feed rate of about $1 \mathrm{t} / \mathrm{h}$. Analysis of the sample showed a content of about $8 \mathrm{~kg}$ of aluminium and $10 \mathrm{~kg}$ of copper-zinc and lead alloys (see Table II). The remaining materials were sinters, stone and glass. The height of the submerged internal was $0.8 \mathrm{~m}$ and it was rotated at a speed of one rotation in $12 \mathrm{~s}$. The material was fed just above the water level using a vibrating feeder and the feeder was positioned so that all material dropped into the water along a single radius of the separation volume (see Fig. 1(b)). The splitter between the first and second compartment was set at $2.0 \mathrm{~s}$ of rotation time measured from the feeding point. Therefore the separator ran at a cut terminal velocity of $0.4 \mathrm{~m} / \mathrm{s}$ between the first and the second compartment (cf. Fig. 5: $-4+2 \mathrm{~mm}$ and $-6+4 \mathrm{~mm}$ graphs). The products from the first two compartments were analysed. The results show that $98 \%$ of the heavy non-ferrous ends up in the first compartment together with less than $2 \%$ of the aluminium and 
stony fractions. Most of the contaminants found in the heavy non-ferrous concentrate were large and smooth, almost spherical particles of stone and aluminium.

The results from this experiment and the data of Fig. 5 suggest that it is possible to separate the entire $-10+2 \mathrm{~mm}$ mixture of non-ferrous scrap into light and heavy alloys by a classification of the material on a bar screen into a $-6+2 \mathrm{~mm}$ fraction and a $-10+6 \mathrm{~mm}$ fraction, followed by a separation on terminal velocity with two feeding points, $2.0 \mathrm{~s}$ and $1.7 \mathrm{~s}$ of rotation time from the splitter, respectively.

\section{CONCLUSION}

Non-ferrous scrap in the range from 2 to $10 \mathrm{~mm}$ can be separated into light and heavy alloys by means of a cheap and simple kinetic separation in water, such that about 1 to $2 \%$ of the light alloys end up in the heavies and vice versa. The same technique is also promising for plastics scrap consisting of polymers with different densities or for mixtures of polymers that are polluted with aluminium from closures or thin foils from labels and barrier layers.

\section{Acknowledgement}

The authors thank Miss Francesca Settimo for the analysis of product samples.

\section{References}

[1] N.L. Weiss, SME Mineral Processing Handbook, AIME, New York, 1985, 3-32.

[2] H. Schubert, Aufbereitung fester Stoffe, Band II: Sortierprozesse, 4th Edn., DVG Stuttgart, 1996, 3-71.

[3] J. Quindt, H. Mueller and A. Tolke, Einfuehrung der Setztechnik in der Beiakkuschrott-Aufbereitung, In: G. Schubert and C. Schoene (Eds.), Proceedings of the 50. Berg- und Huttenmannischen Tag, 1999, Freiberg, Freiberger Forschungshefte A850 Umweltschutz, 1999, pp. 76-85.

[4] P.C. Rem and L.A. van Kooy, Patent Application 1022952, Werkwijze voor het scheiden van deeltjes en inrichting daarvoor [Method and apparatus for separating particles], 2003.

[5] R. H. Perry and D.W. Green, Perry's Chemical Engineering Handbook, 6th Edn., McGraw Hill, New York, pp. 5-64.

\section{BIOGRAPHIES}

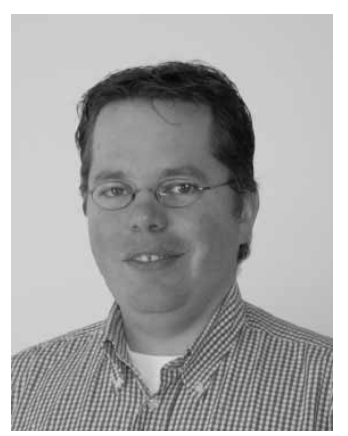

Laurens van Kooy is a researcher at the Resource Engineering section of Delft University of Technology. His graduation project was about locating halogens in plastic waste for reducing toxic combustion products. His present interests are in recycling and separation technology, in particular the separation of bottom ash from municipal waste incinerators. 


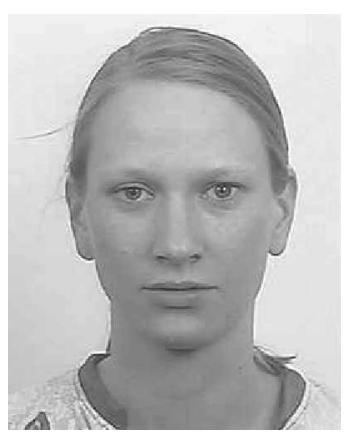

Marieke Mooij is a thesis student at the Resource Engineering section of Delft University. Last year she followed the European Mineral Engineering Course in Delft, Aachen, London and Helsinki. Presently her focus is mainly on recycling and separation technology.

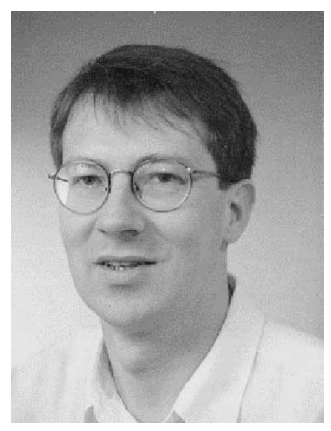

P.C. Rem is a research coordinator for the Resource Engineering Section of Delft University, the Netherlands. He studied physics at Leiden University and did his Ph.D. in the field of technical superconductors at Twente University in the Netherlands, before joining the Shell Research Laboratories in Amsterdam. His present interests are in separation technology. 